$11-1-2003$

\title{
Approximate Bayesian Confidence Intervals For The Variance Of A Gaussian Distribution
}

Vincent A. R. Camara

University of South Florida, gvcamara@ij.net

Follow this and additional works at: http://digitalcommons.wayne.edu/jmasm

Part of the Applied Statistics Commons, Social and Behavioral Sciences Commons, and the Statistical Theory Commons

\section{Recommended Citation}

Camara, Vincent A. R. (2003) "Approximate Bayesian Confidence Intervals For The Variance Of A Gaussian Distribution," Journal of Modern Applied Statistical Methods: Vol. 2 : Iss. 2 , Article 8.

DOI: $10.22237 /$ jmasm/1067645280

Available at: http://digitalcommons.wayne.edu/jmasm/vol2/iss2/8 


\title{
Approximate Bayesian Confidence Intervals For The Variance Of A Gaussian Distribution
}

\author{
Vincent A. R. Camara \\ University of South Florida
}

The aim of the present study is to obtain and compare confidence intervals for the variance of a Gaussian distribution. Considering respectively the square error and the Higgins-Tsokos loss functions, approximate Bayesian confidence intervals for the variance of a normal population are derived. Using normal data and SAS software, the obtained approximate Bayesian confidence intervals will then be compared to the ones obtained with the well known classical method. The Bayesian approach relies only on the observations. It is shown that the proposed approximate Bayesian approach relies only on the observations. The classical method, that uses the Chi-square statistic, does not always yield the best confidence intervals.

Key words: Estimation, loss functions, statistical analysis

\section{Introduction}

There is a significant amount of research in Bayesian analysis and modeling, which has been published the last twenty-five years; see references. A Bayesian analysis implies the exploitation of a suitable prior information and the choice of a loss function in association with Bayes' Theorem. It rests on the notion that a parameter within a model is not merely an unknown quantity but rather behaves as a random variable, which follows some distribution. In the area of life testing, it is indeed realistic to assume that a life parameter is stochastically dynamic. This assertion is supported by the fact that the complexity of electronic and structural systems is likely to cause undetected component interactions resulting in an unpredictable fluctuation of the life parameter. Recently, Drake (1966) gave an excellent account for the use of Bayesian statistics in reliability problems.

Vincent A. R. Camara earned a Ph.D. in Mathematics/Statistics. His research interests are in the theory and applications of Bayesian and empirical Bayes analyses with emphasis on the computational aspect of modeling. He is featured in the 2003 edition of Marquis Who's Who in America. (E-mail: gvcamara@ij.net)
As he pointed out, "He (Bayesian) realizes ... that his selection of a prior (distribution) to express his present state of knowledge will necessarily be somewhat arbitrary. But he greatly appreciates this opportunity to make his entire assumptive structure clear to the world..."

In the present study, we shall consider a classical and useful underlying model. That is, we shall consider the normal underlying model characterized by

$$
\begin{aligned}
& f(x)=\frac{1}{\sqrt{2 \pi} \sigma} e^{-\frac{1}{2}\left(\frac{x-\mu}{\sigma}\right)^{2}} ; \\
& -\infty \prec x \prec \infty,-\infty \prec \mu \prec \infty, \sigma \succ 0 .
\end{aligned}
$$

As we well know, once the underlying model is found to be normally or approximately normally distributed, the classical approach uses the Chi-square statistic and considers the following confidence interval for the population variance $\sigma^{2}$ :

$$
\left[\frac{(n-1) s^{2}}{\chi_{n-1, \alpha / 2}^{2}}, \frac{(n-1) s^{2}}{\chi_{n-1,1-\alpha / 2}^{2}}\right]
$$

For the above model (1), approximate Bayesian confidence bounds for the parameter 
$\sigma^{2}$ will be derived to challenge the classical approach (2). In the study, we shall denote the inverse of the population variance $\sigma^{2}$ by $\theta$ and its corresponding estimate by $\hat{\theta}$.

Although there is no specific analytical procedure that allows us to identify the appropriate loss function to be used, the most commonly used is the square error loss function. One of the reasons for selecting this loss function is because of its analytical tractability in Bayesian analysis. As it will be shown, selecting the square error loss does not always lead to the best approximate Bayesian confidence intervals. However, the obtained approximate Bayesian confidence intervals corresponding to the square error and the Higgins-Tsokos loss functions will be respectively used to challenge the classical method (2). The loss functions that will be used are given below, along with a statement of their key characteristics.

Square error loss function

The popular square error loss function places a small weight on estimates near the true value and proportionately more weight on extreme deviation from the true value of the parameter. Its popularity is due to its analytical tractability in Bayesian modeling. The square error loss is defined as follows:

$$
\left.L_{S E} \stackrel{\wedge}{\theta}, \theta\right)=(\stackrel{\wedge}{\theta}-\theta)^{2}
$$

\section{Higgins-Tsokos loss function}

The Higgins-Tsokos loss function places a heavy penalty on extreme over- or underestimation. That is, it places an exponential weight on extreme errors. The Higgins-Tsokos loss function is defined as follows:

$$
L_{H T}(\hat{\theta}, \theta)=\frac{f_{1} e^{f_{2}(\hat{\theta}-\theta)}+f_{2} e^{-f_{1}(\hat{\theta}-\theta)}}{f_{1}+f_{2}}-1 \text {, }
$$$$
f_{1}, f_{2} \succ 0 \text {. }
$$

We shall assume that $\theta$ behaves as a random variable and is being characterized by the Pareto probability density function given by

$$
f_{1}(\theta)=\frac{a}{b}\left(\frac{b}{\theta}\right)^{a+1} ; \theta \geq b \succ 0, a \succ 0 .
$$

where $\theta=1 / \sigma^{2}$.

The Pareto prior has been selected because of its mathematical tractability. Using observations from normal distributions, we will approximate the Pareto prior (5) in such a way that good approximate Bayesian estimates of $\theta$ are obtained.

Preliminaries

Let $x_{1}, x_{2}, \ldots \ldots, x_{n}$ denote the observations of a given system that are being characterized by the normal distribution. Replacing $1 / \sigma^{2}$ by $\theta$, we obtain the following characterization of the normal underlying model defined in (1).

$$
\begin{aligned}
& f(x)=\frac{1}{\sqrt{2 \pi}} \theta^{\frac{1}{2}} e^{-\theta \frac{(x-\mu)^{2}}{2}} ; \\
& -\infty \prec x \prec \infty, \theta \succ 0
\end{aligned}
$$

This leads to the following posterior distribution:

$$
h(\theta \backslash x) \frac{\theta^{\frac{n}{2}-a-1} e^{-\theta \sum_{1}^{n} \frac{\left(x_{i}-\mu\right)^{2}}{2}}}{\int_{b}^{\infty} \theta^{\frac{n}{2}-a-1} e^{-\theta \sum_{1}^{n} \frac{\left(x_{i}-\mu\right)^{2}}{2}} d \theta}, \theta \succ b .
$$

\section{Methodology}

Approximate Bayesian confidence bounds of $\sigma^{2}$ when the population mean $\mu$ is known.

With respectively the following approximate priors for the square error and the Higgins-Tsokos loss functions, good 
approximate Bayesian estimates of $\theta$ are obtained.

Approximate prior for the square error loss:

$$
\begin{aligned}
\bar{g}(\theta) & =\frac{a 1}{b 1}\left(\frac{b 1}{\theta}\right)^{a 1+1} ; \\
a 1 & =\frac{n}{2}-1, b 1=\frac{n-2}{\sum_{i=1}^{n}\left(x_{i}-\mu\right)^{2}}
\end{aligned}
$$

Approximate prior for the Higgins-Tsokos loss:

$$
\begin{aligned}
& \bar{g}_{1}(\theta)=\frac{a o}{b o}\left(\frac{b o}{\theta}\right)^{a o+1} ; \\
& ; a_{0}=\frac{n}{2}-1, b o=\frac{n-1}{\sum_{i=1}^{n}\left(x_{i}-\mu\right)^{2}}-F(n, \mu)
\end{aligned}
$$

where

$$
F(n, \mu)=\frac{1}{f_{1}+f_{2}} \operatorname{Ln}\left(\frac{\sum_{i=1}^{n} \frac{\left(x_{i}-\mu\right)^{2}}{2}+f_{2}}{\sum_{i=1}^{n} \frac{\left(x_{i}-\mu\right)^{2}}{2}-f_{1}}\right)
$$

with

$$
f_{1} \prec \sum_{i=1}^{n} \frac{\left(x_{i}-\mu\right)^{2}}{2}
$$

It's easily shown that the approximate Bayesian estimate of the parameter $\theta$, subject to the square error loss, is the same as the Bayesian estimate of $\theta$ under the HigginsTsokos loss. They are equal to

$$
\frac{n-1}{\sum_{i=1}^{n}\left(x_{i}-\mu\right)^{2}}
$$

Using respectively the approximate posterior distributions that correspond to (8) and (9), along with the equalities $P(\theta \succ L \mid x)=1-\alpha / 2$ and $P(\theta \succ U \mid x)=\alpha / 2$, we respectively obtain the following lower and upper confidence bounds for $\theta$ :

Approximate Bayesian confidence bounds of $\theta$ corresponding to the square error loss function when $\mu$ is known:

$$
\begin{gathered}
L_{\theta(S E)}=\frac{n-2-2 \operatorname{Ln}(1-\alpha / 2)}{\sum_{i=1}^{n}\left(x_{i}-\mu\right)^{2}} \\
U_{\theta(S E)}=\frac{n-2-2 \operatorname{Ln}(\alpha / 2)}{\sum_{i=1}^{n}\left(x_{i}-\mu\right)^{2}}
\end{gathered}
$$

Approximate Bayesian confidence bounds of $\theta$ corresponding to the Higgins-Tsokos loss function when $\mu$ is known:

$$
\begin{gathered}
L_{\theta(H T)}=\frac{n-1-2 \operatorname{Ln}(1-\alpha / 2)}{\sum_{i=1}^{n}\left(x_{i}-\mu\right)^{2}}-F(n, \mu) \\
U_{\theta(H T)}=\frac{n-1-2 \operatorname{Ln}(\alpha / 2)}{\sum_{i=1}^{n}\left(x_{i}-\mu\right)^{2}}-F(n, \mu)
\end{gathered}
$$

Thus when the population mean is known, (10) and (11) respectively yield the following $100(1-\alpha) \% \quad$ approximate Bayesian confidence bounds for the normal population variance $\sigma^{2}$ :

Confidence bounds corresponding to the square error loss: 


$$
\begin{gathered}
L_{\sigma^{2}(S E)}=\frac{\sum_{i=1}^{n}\left(x_{i}-\mu\right)^{2}}{n-2-2 \ln (\alpha / 2)} \\
U_{\sigma^{2}(S E)}=\frac{\sum_{i=1}^{n}\left(x_{i}-\mu\right)^{2}}{n-2-2 \ln (1-\alpha / 2)},
\end{gathered}
$$

Confidence bounds corresponding to the Higgins-Tsokos loss:

$$
\begin{aligned}
L_{\sigma^{2}(H T)} & =\frac{1}{\frac{n-1-2 \operatorname{Ln}(\alpha / 2)}{\sum_{i=1}^{n}\left(x_{i}-\mu\right)^{2}}-F(n, \mu)} \\
U_{\sigma^{2}(H T)} & =\frac{1}{\frac{n-1-2 \operatorname{Ln}(1-\alpha / 2)}{\sum_{i=1}^{n}\left(x_{i}-\mu\right)^{2}}-F(n, \mu)} .
\end{aligned}
$$

Approximate Bayesian confidence bounds of $\sigma^{2}$ when the population mean $\mu$ is unknown.

In the case where the population mean $\mu$ is unknown, it is estimated by the sample mean $\bar{X}$ and we obtain the following:

Approximate Bayesian confidence bounds of $\theta$ corresponding to the square error loss function when $\mu$ is unknown:

$$
\begin{gathered}
L_{\theta(S E)}=\frac{n-2-2 \operatorname{Ln}(1-\alpha / 2)}{\sum_{i=1}^{n}\left(x_{i}-\bar{x}\right)^{2}} \\
U_{\theta(S E)}=\frac{n-2-2 \operatorname{Ln}(\alpha / 2)}{\sum_{i=1}^{n}\left(x_{i}-\bar{x}\right)^{2}}
\end{gathered}
$$

Approximate Bayesian confidence bounds of $\theta$ corresponding to the Higgins-Tsokos loss function when $\mu$ is unknown:

$$
\begin{gathered}
L_{\theta(H T)}=\frac{n-1-2 \operatorname{Ln}(1-\alpha / 2)}{\sum_{i=1}^{n}\left(x_{i}-\bar{x}\right)^{2}}-F(n, \bar{x}) \\
U_{\theta(H T)}=\frac{n-1-2 \operatorname{Ln}(\alpha / 2)}{\sum_{i=1}^{n}\left(x_{i}-\bar{x}\right)^{2}}-F(n, \bar{x}) .
\end{gathered}
$$

Thus when $\mu$ is unknown (14) and (15) respectively yield the following $100(1-\alpha) \% \quad$ approximate Bayesian confidence bounds for the normal population variance $\sigma^{2}$ :

Confidence bounds corresponding to the square error loss:

$$
\begin{aligned}
L_{\sigma^{2}(S E)} & =\frac{\sum_{i=1}^{n}\left(x_{i}-\bar{x}\right)^{2}}{n-2-2 \ln (\alpha / 2)} \\
U_{\sigma^{2}(S E)} & =\frac{\sum_{i=1}^{n}\left(x_{i}-\bar{x}\right)^{2}}{n-2-2 \ln (1-\alpha / 2)},
\end{aligned}
$$

Confidence bounds corresponding to the Higgins-Tsokos loss:

$$
\begin{aligned}
L_{\sigma^{2}(H T)} & =\frac{1}{\frac{n-1-2 \operatorname{Ln}(\alpha / 2)}{\sum_{i=1}^{n}\left(x_{i}-\bar{x}\right)^{2}}-F(n, \bar{x})} \\
U_{\sigma^{2}(H T)} & =\frac{1}{\frac{n-1-2 \operatorname{Ln}(1-\alpha / 2)}{\sum_{i=1}^{n}\left(x_{i}-\bar{x}\right)^{2}}-F(n, \bar{x})} .
\end{aligned}
$$

Results

In order to compare the proposed approximate Bayesian approach to the classical method, samples that have been obtained from normally distributed populations (Examples 1, 2, 3, .4, 7) as well as approximately normal populations (Examples 5, 6) will be considered. SAS software is used to obtain the normal population parameters $\mu$ and $\sigma$ 
corresponding to each of the examples. The proposed approximate Bayesian estimates of the variance (16) (17) will be used. For the Higgins-Tsokos loss function, we will consider $f_{1}=1, f_{2}=1$. The lengths of the classical and approximate Bayesian confidence intervals are respectively denoted by $l_{C}, l_{S E}$ and $l_{H T}$.

Example 1. (Data obtained from Prem S. Mann, Introductory Statistics, Third edition, page 504,1998$)$.

24, 28, 22, 25, 24, 22, 29, 26, 25, 28, 19, 29. Normal population distribution obtained with SAS: $N(\mu=25.083, \sigma=3.1176)$ Population and sample variances: $\sigma^{2}=9.71943$, $s^{2}=9.719696$.

Table 1. Classical and approximate Bayesian confidence intervals of $\sigma^{2}$ corresponding to the first data set.

\begin{tabular}{|l|l|l|l|}
\hline $\begin{array}{l}\text { C L. } \\
\% .\end{array}$ & $\begin{array}{l}\text { Classical } \\
\text { Bounds }\end{array}$ & $\begin{array}{c}\text { Approx.Bayes. } \\
\text { Bounds (SE) }\end{array}$ & $\begin{array}{c}\text { Approx.Bayes. } \\
\text { Bounds (HT) }\end{array}$ \\
\hline 80 & $6.18-$ & $7.32-10.47$ & $8.08-10.23$ \\
\hline 90 & $5.43-$ & $6.68-10.58$ & $7.32-10.47$ \\
& 23.36 & & \\
\hline 95 & $4.87-$ & $6.15-10.63$ & $6.68-10.58$ \\
& 28.01 & & \\
\hline 99 & $3.99-$ & $5.19-10.68$ & $5.56-10.67$ \\
& 41.07 & & \\
\hline
\end{tabular}

\begin{tabular}{|c|c|c|}
\hline $\begin{array}{c}\text { Confidence } \\
\text { level }\end{array}$ & $\left(l_{C}\right) \div\left(l_{S E}\right)$ & $\begin{array}{c}\left(l_{C}\right) \div \\
\left(l_{H T}\right)\end{array}$ \\
\hline $80 \%$ & 4.1193 & 6.0455 \\
\hline $90 \%$ & 4.6021 & 5.6927 \\
\hline $95 \%$ & 5.1589 & 5.9373 \\
\hline $99 \%$ & 6.7538 & 7.2636 \\
\hline
\end{tabular}

Example 2. Data obtained from Prem S. Mann, Introductory Statistics, Third edition, page 504, 1998.

13, 11, 9, 12, 8, 10, 5, 10, 9, 12, 13.

$\begin{array}{llr}\text { Normal } & \text { population } & \text { distribution } \\ \text { obtained } & \text { with } & \text { SAS: }\end{array}$ $N(\mu=10.182, \sigma=2.4008)$. Population and sample variances: $\quad \sigma^{2}=5.76384$, $s^{2}=5.763636$.

Table 2: Classical and approximate Bayesian confidence intervals of $\sigma^{2}$ corresponding to the second data set.

\begin{tabular}{|c|c|c|c|}
\hline $\begin{array}{l}\text { C L. } \\
\% \text {. }\end{array}$ & $\begin{array}{l}\text { Classical } \\
\text { Bounds }\end{array}$ & $\begin{array}{l}\text { Approx.Bayes. } \\
\text { Bounds (SE) }\end{array}$ & $\begin{array}{l}\text { Approx.Bayes. } \\
\text { Bounds (HT) }\end{array}$ \\
\hline 80 & $\begin{array}{l}3.60- \\
11.84\end{array}$ & $4.23-6.25$ & $4.71-6.10$ \\
\hline 90 & $\begin{array}{l}3.14- \\
14.62\end{array}$ & $3.84-6.33$ & $4.23-6.25$ \\
\hline 95 & $\begin{array}{l}2.81- \\
17.75\end{array}$ & $3.51-6.36$ & $3.84-6.33$ \\
\hline 99 & $\begin{array}{l}2.28- \\
26.73\end{array}$ & $2.94-6.39$ & $3.16-6.38$ \\
\hline \multicolumn{2}{|c|}{$\begin{array}{c}\text { Confidence } \\
\text { level }\end{array}$} & $\begin{array}{c}\left(l_{C}\right) \div \\
\left(l_{S E}\right)\end{array}$ & $\begin{array}{l}\left(l_{C}\right) \div \\
\left(l_{H T}\right)\end{array}$ \\
\hline \multicolumn{2}{|c|}{$80 \%$} & 4.0777 & 5.9530 \\
\hline \multicolumn{2}{|c|}{$90 \%$} & 4.6157 & 5.6804 \\
\hline \multicolumn{2}{|c|}{$95 \%$} & 5.2426 & 6.0051 \\
\hline \multicolumn{2}{|c|}{$99 \%$} & 7.0734 & 7.5801 \\
\hline
\end{tabular}

Example 3. Data obtained from Prem S. Mann, Introductory Statistics, Third edition, page 504, 1998.

$16,14,11,19,14,17,13,16,17,18,19,12$. 
Normal population distribution obtained with SAS: $N(\mu=15.5, \sigma=2.6799)$. Population and sample variances: $\sigma^{2}=7.18186$, $s^{2}=7.181818$.

Table 3. Classical and approximate Bayesian confidence intervals of $\sigma^{2}$ corresponding to the third data set.

\begin{tabular}{|l|l|l|l|}
\hline $\begin{array}{l}\text { C L. } \\
\% .\end{array}$ & $\begin{array}{l}\text { Classical } \\
\text { Bounds }\end{array}$ & $\begin{array}{c}\text { Approx.Bayes. } \\
\text { Bounds (SE) }\end{array}$ & $\begin{array}{l}\text { Approx.Bayes. } \\
\text { Bounds (HT) }\end{array}$ \\
\hline 80 & $4.57-$ & $5.40-7.73$ & $5.97-7.56$ \\
& 14.16 & & \\
\hline 90 & $4.01-$ & $4.94-7.81$ & $5.40-7.73$ \\
& 17.26 & & \\
\hline 95 & $3.60-$ & $4.54-7.86$ & $4.94-7.81$ \\
& 20.70 & & \\
\hline 99 & $2.95-$ & $3.83-7.89$ & $4.11-7.88$ \\
& 30.34 & & \\
\hline
\end{tabular}

\begin{tabular}{|c|c|c|}
\hline $\begin{array}{c}\text { Confidence } \\
\text { level }\end{array}$ & $\left(l_{C}\right) \div\left(l_{S E}\right)$ & $\left(\begin{array}{c}\left(l_{C}\right) \div \\
\left(l_{H T}\right)\end{array}\right.$ \\
\hline $80 \%$ & 4.1194 & 6.0456 \\
\hline $90 \%$ & 4.6022 & 5.6926 \\
\hline $95 \%$ & 5.1592 & 5.9375 \\
\hline $99 \%$ & 6.7539 & 7.2636 \\
\hline
\end{tabular}

Example 4. Data obtained from Prem S. Mann, Introductory Statistics, Third edition, page 504, 1998.

27, 31, 25, 33, 21, 35, 30, 26, 25,31.33.30, 28.

Normal population distribution obtained with SAS: $N(\mu=28.846, \sigma=3.9549)$. Population and sample variances: $\quad \sigma^{2}=15.64123$, $s^{2}=15.641025$.
Table 4. Classical and approximate Bayesian confidence intervals of $\sigma^{2}$ corresponding to the fourth data set.

\begin{tabular}{|l|c|c|c|}
\hline C L. & Classical & Approx.Bayes. & Approx.Bayes. \\
& Bounds & Bounds (SE) & Bounds (HT) \\
\hline 80 & 10.11 & $12.02-$ & $13.20-$ \\
& 29.77 & 16.74 & 16.39 \\
\hline 90 & $8.92-$ & $11.04-$ & $12.02-$ \\
& 35.91 & 16.90 & 16.74 \\
\hline 95 & $8.04-$ & $10.21-$ & $11.04-$ \\
& 42.61 & 16.98 & 16.90 \\
\hline 99 & $6.63-$ & $8.69-$ & $9.28-$ \\
& 61.05 & 17.04 & 17.03 \\
\hline
\end{tabular}

\begin{tabular}{|c|c|c|}
\hline Confidence level & $\left(l_{C}\right) \div\left(l_{S E}\right)$ & $\left(l_{C}\right) \div$ \\
& & $\left(l_{H T}\right)$ \\
\hline $80 \%$ & 4.1688 & 6.1471 \\
\hline $90 \%$ & 4.6063 & 5.7243 \\
\hline $95 \%$ & 5.1059 & 5.9013 \\
\hline $99 \%$ & 6.5129 & 7.0273 \\
\hline
\end{tabular}

Example 5. Data obtained from James T. McClave/Terry Sincich A first course in Statistics, page 301, Sixth edition, 1997

\section{2, 33, 42, 44, 41, 50, 44, 51, 45, 38,} 37,40,44, 50, 43.

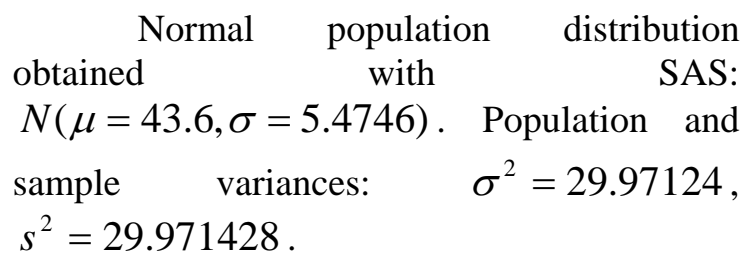


Table 5. Classical and approximate Bayesian confidence intervals of $\sigma^{2}$ corresponding to the fifth data set.

\begin{tabular}{|l|c|c|c|}
\hline $\begin{array}{l}\text { C L. } \\
\% .\end{array}$ & Classical & $\begin{array}{c}\text { Approx.Bayes. } \\
\text { Bounds }\end{array}$ & $\begin{array}{c}\text { Approx.Bayes. } \\
\text { Bounds (HE) }\end{array}$ \\
\hline 80 & $19.92-$ & $23.83-31.76$ & $25.87-$ \\
& 53.86 & & 31.20 \\
\hline 90 & 17.71 & $22.09-32.02$ & $23.83-$ \\
& - & & 31.76 \\
\hline 95 & $16.06-$ & $20.59-32.15$ & $22.09-$ \\
& 74.54 & & 32.02 \\
\hline 99 & $13.39-$ & $17.78-32.25$ & $18.89-$ \\
& 102.96 & & 32.22 \\
\hline
\end{tabular}

\begin{tabular}{|c|c|c|}
\hline $\begin{array}{c}\text { Confidence } \\
\text { level }\end{array}$ & $\left(l_{C}\right) \div\left(l_{S E}\right)$ & $\left(\begin{array}{c}\left(l_{C}\right) \div \\
\left(l_{H T}\right)\end{array}\right.$ \\
\hline $80 \%$ & 4.2814 & 6.3629 \\
\hline $90 \%$ & 4.6465 & 5.8198 \\
\hline $95 \%$ & 5.0583 & 5.8889 \\
\hline $99 \%$ & 6.1902 & 6.7170 \\
\hline
\end{tabular}

Example 6. Data obtained from James $\mathrm{T}$. McClave/Terry Sincich A first course in Statistics, page 301, Sixth edition, 1997.

$52,43,47,56,62,53,61,50,56,52$, 53, 60, 50, 48, 60, 55.

$$
\begin{aligned}
& \text { Normal } \begin{array}{c}
\text { population } \\
\text { with }
\end{array} \\
& \begin{array}{l}
\text { distribution } \\
\text { obtained }
\end{array} \\
& N(\mu=53.625, \sigma=5.4145) \text {. Population and } \\
& \text { sample variances: } \quad \sigma^{2}=29.31681 \text {, } \\
& s^{2}=29.316666 \text {. }
\end{aligned}
$$

Table 6. Classical and approximate Bayesian confidence intervals of $\sigma^{2}$ corresponding to the sixth data set.

\begin{tabular}{|l|l|c|c|}
\hline C L. & Classical & Approx.Bayes & $\begin{array}{c}\text { Approx.Bayes. } \\
\text { Bounds (HT) }\end{array}$ \\
\hline 80 & $19.71-$ & $23.63-$ & $25.53-30.44$ \\
& 51.45 & 30.94 & \\
\hline 90 & $17.59-$ & $21.99-$ & $23.63-30.94$ \\
& 60.56 & 31.18 & \\
\hline 95 & $15.99-$ & $20.57-$ & $21.99-31.18$ \\
& 70.22 & 31.29 & \\
\hline 99 & $13.40-$ & $17.87-$ & $18.94-31.36$ \\
& 95.57 & 31.38 & \\
\hline
\end{tabular}

\begin{tabular}{|c|c|c|}
\hline $\begin{array}{c}\text { Confidence } \\
\text { level }\end{array}$ & $\left(l_{C}\right) \div\left(l_{S E}\right)$ & $\left(\begin{array}{c}\left(l_{C}\right) \div \\
\left(l_{H T}\right)\end{array}\right.$ \\
\hline $80 \%$ & 4.3422 & 6.4743 \\
\hline $90 \%$ & 4.6781 & 5.8754 \\
\hline $95 \%$ & 5.0551 & 5.9036 \\
\hline $99 \%$ & 6.0822 & 6.6163 \\
\hline
\end{tabular}

Example 7. The following observations have been obtained from the collection of SAS data sets.

50, 65, 100, 45, 111, 32, 45, 28, 60, 66, 114, 134, 150, 120, 77, 108, 112, 113,80,77, 69, 91, 116, 122, 37, 51, 53, 131, 49, 69, 66, 46, 131, 103, 84, 78.

$\begin{array}{ccr}\text { Normal population } & \text { distribution } \\ \text { obtained } & \text { with } & \text { SAS: }\end{array}$ $N(\mu=82.861, \sigma=33.226)$. Population and sample variances: $\quad \sigma^{2}=1103.96716$, $s^{2}=1103.951587$. 
Table 7: Classical and approximate Bayesian confidence intervals of $\sigma^{2}$ corresponding to the seventh data set.

\begin{tabular}{|c|c|c|c|}
\hline C L. & Classical & $\begin{array}{c}\text { Approx.Bayes. } \\
\text { Bounds (SE) }\end{array}$ & $\begin{array}{c}\text { Approx.Bayes. } \\
\text { Bounds (HT) }\end{array}$ \\
\hline 80 & $839.4-$ & $1000.8-$ & $1038.1-$ \\
1556.4 & 1129.4 & 1121.6 \\
\hline 90 & $776.4-$ & $966.1-$ & $1000.8-$ \\
& 1717.1 & 1133.0 & 1129.4 \\
\hline 95 & $726.8-$ & $933.7-$ & $966.1-$ \\
& 1874.5 & 1134.7 & 1133.0 \\
\hline 99 & $641.6-$ & $866.3-$ & $894.1-$ \\
& 2240.2 & 1136.0 & 1135.7 \\
\hline
\end{tabular}

\begin{tabular}{|c|c|c|}
\hline $\begin{array}{c}\text { Confidence } \\
\text { level }\end{array}$ & $\begin{array}{c}\left(l_{C}\right) \div \\
\left(l_{S E}\right)\end{array}$ & $\begin{array}{c}\left(l_{C}\right) \div \\
\left(l_{H T}\right)\end{array}$ \\
\hline $80 \%$ & 5.5772 & 8.5808 \\
\hline $90 \%$ & 5.6388 & 7.3176 \\
\hline $95 \%$ & 5.7119 & 6.8792 \\
\hline $99 \%$ & 5.9277 & 6.6181 \\
\hline
\end{tabular}

All seven Tables show that the proposed approximate Bayesian confidence intervals contain the population variance $\sigma^{2}$. Also, the lengths of the obtained classical confidence intervals are more than four times greater than the ones corresponding to the proposed approach.

\section{Conclusion}

In the present study, approximate Bayesian confidence intervals for the variance of a normal population under two different loss functions have been derived. The loss functions that are employed are the square error and the Higgins-Tsokos loss functions. Based on the above numerical results we can conclude the following:

The classical method used to construct confidence intervals for the variance of a normal population does not always yield the best coverage accuracy. In fact, each of the obtained approximate Bayesian confidence intervals contains the population variance and is strictly included in the corresponding confidence interval obtained with the classical method.

Contrary to the classical method that uses the Chi-square statistic, the proposed approach relies only on the observations.

With the proposed approach, approximate Bayesian confidence intervals for a normal population variance are easily computed for any level of significance.

The approximate Bayesian approach under to the popular square error loss function does not always yield the best approximate Bayesian results. In fact, the Higgins-Tsokos loss function performs better in the above examples.

Bayesian analysis contributes to reinforcing well-known statistical theories such as the estimation theory.

\section{References}

Bhattacharya, S. K. (1967). Bayesian approach to life testing and reliability estimation. Journal American Statistical Association, 62, 48-62.

Bernard, H. (1976). A survey of statistical methods in system reliability using Bernoulli sampling of components. Proceedings of the conference on the theory and applications of Reliability with emphasis on Bayesian and Nonparametric Methods. NY: Academic.

Britney, R. R., \& Winkler, R. L. (1968). Bayesian III point estimation under various loss functions. American Statistical Association, 356-364.

Camara, V. A. R., \& Tsokos, C. P. (1996). Effect of Loss Functions on Bayesian Reliability Analysis. Proceedings of International Conference on Nonlinear Problems in Aviation and Aerospace, 75-90.

Camara, V. A. R., \& Tsokos, C. P. (1998). Bayesian reliability modeling with applications. UMI Publishing Company. 
Camara, V. A. R., \& Tsokos, C. P. (1999). Bayesian estimate of a parameter and choice of the loss function. Nonlinear Studies Journal, 6, 55-64.

Camara, V. A. R., \& Tsokos, C. P. (1999). The effect of loss functions on empirical bayes reliability analysis. Journal of Engineering Problems, Boston University, http://bujep.bu.edu.

Camara, V. A. R. (2002). Approximate Bayesian confidence intervals for the variance of a Gaussian distribution. 2002 Proceedings of the American Statistical Association, Statistical Computing Section New York, NY: American Statistical Association.

Canfield, R. V. (1970). A Bayesian approach to reliability estimation using a loss function. IEEE Trans. Reliability R-19 (1), 1316.

Vincent A. R. Camara \& Tsokos, C. P. (1999, November). Sensitivity behavior of Bayesian reliability analysis for different loss functions. International Journal of Applied Mathematics.

Drake, A. W. (1966). Bayesian statistics for the reliability eng. proc. Annual Symposium on Reliability, 315-320.

Higgins, J. J., \& Tsokos, C. P. (1976). Comparison of Bayes estimates of failure intensity for fitted priors of life data. Proceedings of the Conference on the Theory and Applications of Reliability with Emphasis on Bayesian and Nonparametric Methods. NY: Academic.

Higgins, J. J. \& Tsokos, C. P. (1976). On the behavior of some quantities used in Bayesian reliability demonstration tests. IEEE Trans. Reliability R-25, (4), 261-264.
Higgins, J. J., \& Tsokos, C. P. (1980). A study of the effect of the loss function on bayes estimates of failure intensity, MTBF, and reliability. Applied Mathematics and Computation, 6, 145-166.

McClave, J. T., \& Sincich, T. A. (1997). First course in statistics $\left(6^{\text {th }}\right.$ ed.). Upper Saddle River, NJ: Prentice Hall.

Mann, P. S. (1998), Introductory Statistics, $\left(3^{\text {rd }}\right.$ ed). NY: Wiley.

Schafer, et al. (1970). Bayesian reliability demonstration, Phase I Data for the a prior distribution. Rome Air Development Center, Griffis AFBNY RADC-TR-69-389.

Schafer, et al. (1971). Bayesian reliability, Phase II- Development of a priori distribution. Rome Air Development Center, Griffis AFR, NY RADC-YR-71-209.

Schafer, et al. (1973). Bayesian reliability demonstration Phase III Development of test plans, Rome Air development Center, Griffs AFB, NY RADCTR-73-39.

Shafer, R. E., \& Feduccia, A. J. (1972). Prior distribution fitted to observed reliability data. IEEE Trans. Reliability $R$-21, (3), 148-154

Tsokos, C. P. \& Shimi, I. (Eds). (1976). Proceedings of the Conference on the theory and Applications of Reliability with Emphasis on Bayesian and Nonparametric Methods, Methods, I, II. NY: Academic.

Winkler, R. L. (1972). Introduction to Bayesian inference and decision-making. NY: Holt, Rinehart and Winston. 